\title{
Aberrant expression of miR-483-5p in patients with asymptomatic carotid artery stenosis and its predictive value for cerebrovascular event occurrence
}

\author{
RAN LI $^{1 *}$, LI JIANG $^{2 *}$ and XIAOLING WANG ${ }^{3}$ \\ ${ }^{1}$ The Fourth Department of Encephalopathy, Weifang Hospital of Traditional Chinese Medicine, \\ Weifang, Shandong 261041; ${ }^{2}$ Brain Center, Sunshine Union Hospital, Weifang, Shandong 261000; \\ ${ }^{3}$ Department of Neurology, Liaocheng People's Hospital, Liaocheng, Shandong 252000, P.R. China
}

Received January 6, 2021; Accepted June 18, 2021

DOI: $10.3892 /$ etm.2021.10536

\begin{abstract}
MicroRNAs (miRNAs/miRs) may be used as novel promising diagnostic and prognostic biomarkers for various diseases, including asymptomatic carotid artery stenosis (ACAS). The present study aimed to investigate the abnormal expression of microRNA-483-5p (miR-483-5p) in patients with ACAS and to evaluate its diagnostic value for ACAS screening and its predictive value for cerebrovascular events. A total of 128 patients with ACAS and 76 healthy controls were included in the present study. The expression of miR-483-5p in serum was measured by reverse transcription-quantitative PCR. Receiver operating characteristic (ROC) curve analysis was used to evaluate the diagnostic value of miR-483-5p in patients with ACAS. Kaplan-Meier curves were drawn and Cox regression analysis was used to determine the predictive value of miR-483-5p for cerebrovascular events in patients with ACAS. Serum miR-483-5p levels were significantly increased in patients with ACAS as compared with those in healthy controls. The expression of miR-483-5p was significantly associated with diabetes $(\mathrm{P}=0.011)$, dyslipidemia $(\mathrm{P}=0.047)$ and the degree of carotid stenosis $(\mathrm{P}=0.006)$ in patients with ACAS. In addition, the area under the ROC curve was 0.910 , with a sensitivity of $80.5 \%$ and a specificity of $89.5 \%$ at the cutoff value of 0.705 , indicating that serum miR-483-5p expression has a certain diagnostic value in patients with ACAS. Furthermore, the patients with high miR-483-5p expression had a higher proportion of cerebrovascular events than patients with low miR-483-5p levels
\end{abstract}

Correspondence to: Dr Xiaoling Wang, Department of Neurology, Liaocheng People's Hospital, 45 Huashan Road, Liaocheng, Shandong 252000, P.R. China

E-mail: wangxl@lchospital.cn

*Contributed equally

Key words: microRNA-483-5p, asymptomatic carotid artery stenosis, diagnosis, prognosis, transient ischemic attacks (log-rank $\mathrm{P}=0.011$ ) and miR-483-5p was an independent prognostic marker for predicting the occurrence of cerebrovascular events in patients with ACAS. The results indicated that miR-483-5p expression is significantly increased in patients with ACAS and that abnormal miR-483-5p expression may be a candidate biomarker for ACAS diagnosis and the prediction of cerebrovascular event occurrence.

\section{Introduction}

The carotid artery is one of the main blood vessels supplying the brain, and carotid artery stenosis (CAS) may lead to symptoms of cerebral ischemia. Cerebrovascular events seriously affect human life, health and safety, mainly including transient ischemic events (TIA) and stroke. CAS is a risk factor for cerebrovascular events and is frequently caused by atherosclerosis $(1,2)$. The symptoms of TIA are typically of short duration, unspecific and easily misinterpreted or ignored by both patients and physicians. In addition, $20 \%$ of patients with ischemic stroke develop TIA within hours to days prior to stroke (3). Therefore, it is important to identify the patients with CAS at high risk of TIA or other cerebrovascular events early. CAS is divided into two types, symptomatic CAS and asymptomatic CAS (ACAS), based on the presence or absence of symptoms, respectively (4). As patients with ACAS do not exhibit any typical symptoms, they have a high risk of cerebrovascular events. At present, numerous preventive or therapeutic measures for CAS are in use, such as endarterectomy, stent implantation (5) and drug therapy (1). However, for patients with ACAS, CAS surgery is controversial (6). Furthermore, patients with ACAS treated with statins and antiplatelet drugs were observed to have a certain risk of stroke (7). Therefore, it is important to identify markers for early diagnosis of ACAS and the prediction of cerebrovascular events such as TIA and stroke.

MicroRNAs (miRNAs/miRs) are a class of small non-coding RNAs that have functional roles by regulating their target genes (8). In addition, miRNAs serve as attractive targets for prognostication and therapeutic applications due to their frequent dysregulation in disease (9). It has been indicated that miRNAs are closely related to human diseases, such as 
Alzheimer's disease (10), periodontal disease (11) and cardiovascular disease (12). Abnormal expression of miRNAs, such as miR-106b-5p (13) and miR-92a (14), has been detected in association with the physiological and pathological processes of ACAS, suggesting that miRNAs may be involved in ACAS. Early studies suggested that miR-483-5p has an inhibitory effect on angiogenesis (15), and angiogenesis is an important factor in the initiation and progression of atherosclerosis. In addition, miR-483-5p, a possible stroke-associated miRNA, was upregulated in patients with ischemic stroke (16). Its expression was significantly associated with obesity and diabetes mellitus, which are risk factors for the development of CAS (17). However, the role of miR-483-5p in processes of ACAS has remained elusive.

Therefore, the present study was performed to analyze the expression of miR-483-5p in patients with ACAS, and investigate its diagnostic value for ACAS and its predictive value for cerebrovascular events.

\section{Materials and methods}

Patients and sample collection. The present clinical study was approved by the Ethics Committee of Weifang Hospital of Traditional Chinese Medicine (Weifang, China) and written informed consent was obtained from each participant prior to blood sampling. The present study included 128 patients with ACAS who visited Weifang Hospital of Traditional Chinese Medicine (Weifang, China) between March 2012 and May 2015. All patients underwent color Doppler ultrasound examination and the degree of carotid stenosis was calculated according to the ultrasound results. The included patients with ACAS met the following criteria: i) Ipsilateral internal carotid artery with $>50 \%$ stenosis; ii) age $>18$ years; iii) no history of TIA or stroke; iv) no malignant tumors and no history of malignant tumors; and v) not currently pregnant. In addition, 76 healthy controls matched with the patients with ACAS by age and sex were recruited from a cohort of periodic health examination participants in the same hospital during the same time period. All healthy controls were free of cerebrovascular disease, cardiovascular disease, chronic inflammatory disease, metabolic disease, pregnancy or lactation. Blood samples were drawn from each subject and serum was collected by centrifugation and stored at $-80^{\circ} \mathrm{C}$ for future use.

Data collection. The demographic and clinicopathological characteristics of the participants were recorded on admission, including age, sex, body mass index (BMI), total cholesterol (TC), triglycerides (TG), low-density lipoprotein cholesterol (LDL-C), high-density lipoprotein cholesterol (HDL-C) and fasting plasma glucose (FPG). The presence of diabetes, hypertension, dyslipidemia and degree of carotid stenosis in patients with ACAS were also detected.

Follow-up for cerebrovascular event occurrence. All patients were followed up for 5 years. The occurrence of cerebrovascular events, including TIA, stroke and sudden death, was recorded. Patients who died of other unrelated causes were excluded from the study. The diagnosis of stroke and TIA was according to the American Heart Association/American Stroke Association guidelines (18).
$R N A$ extraction and reverse transcription-quantitative $P C R$ $(R T-q P C R)$. Total RNA in the serum of each subject was extracted using TRIzol ${ }^{\circledR}$ reagent (Invitrogen; Thermo Fisher Scientific, Inc.) following the manufacturer's protocol. The purity and concentration of RNA were confirmed using a NanoDrop ${ }^{\circledR} 2000$ (Thermo Fisher Scientific, Inc.). The obtained RNA was then used for reverse transcription (RT) to synthesize cDNA with the PrimeScript ${ }^{\mathrm{TM}} \mathrm{RT}$ reagent kit (Takara Bio, Inc.). The reaction conditions for reverse transcription are as follows: $42^{\circ} \mathrm{C}$ for $30 \mathrm{~min}$ and $85^{\circ} \mathrm{C}$ for $5 \mathrm{sec}$. The expression level of miR-483-5p was measured using qPCR, which was performed on a 7300 Real-Time PCR System (Applied Biosystems; Thermo Fisher Scientific, Inc.) by using SYBR Green PCR Master Mix kit (Invitrogen; Thermo Fisher Scientific, Inc.). The thermocycling conditions were: Initial denaturation at $95^{\circ} \mathrm{C}$ for $10 \mathrm{~min}$, followed by 40 cycles of $95^{\circ} \mathrm{C}$ for $30 \mathrm{sec}, 60^{\circ} \mathrm{C}$ for $30 \mathrm{sec}$ and $72^{\circ} \mathrm{C}$ for $10 \mathrm{sec}$. U6 was used as an internal control. The primers were as follows: miR-483-5p forward, 5'-GCCGAGAAGACGGGAGGAAA-3' and reverse, 5'-CTCAACTGGTGTCGTGGA-3'; U6 forward, 5'-CTCGCT TCGGCAGCACA-3' and reverse, 5'-AACGCTTCACGAATT TGCGT-3'. The expression of miR-483-5p was calculated using the $2^{-\Delta \Delta C q}$ method (19) and normalized to U6. All procedures were performed following the manufacturers' protocols.

Statistical analysis. All statistical analyses were performed using SPSS 21.0 software (IBM Corp.) and GraphPad Prism 7.0 (GraphPad Software Inc.). Values are expressed as the mean \pm standard deviation. Comparisons between groups were performed using unpaired Student's t-test or the stest. Receiver operating characteristic (ROC) curve analysis was used to determine the diagnostic value of miR-483-5p to distinguish between healthy controls and patients with ACAS. Kaplan-Meier (KM) curves and the log-rank test were used to analyze the association between miR-483-5p levels and cerebrovascular events and the prognostic value of miR-483-5p in predicting the occurrence of cerebrovascular events was assessed by logistic regression using the Cox proportional hazards model. $\mathrm{P}<0.05$ was considered to indicate statistical significance.

\section{Results}

Baseline characteristics of the study cohort. All of the participants were divided into a healthy control group $(n=76)$ and ACAS patient group $(n=128)$. The baseline characteristics are provided in Table I. The healthy control group included 49 males and 27 females with an average age of $59.72 \pm 7.65$ years old. The patient group included 86 males and 42 females with an average age of $61.19 \pm 9.27$ years old. No significant differences were determined between patients with ACAS and healthy controls in terms of age, sex, BMI and HDL-C (all P>0.05). However, compared with the healthy controls, the patients with ACAS exhibited significantly increased levels of TC, TG, LDL-C and FPG (all P<0.05).

Expression of serum miR-483-5p in patients with ACAS. As presented in Fig. 1, the expression of miR-483-5p was detected in the serum of healthy controls and patients with ACAS by RT-qPCR. The expression of miR-483-5p was determined 
Table I. Baseline characteristics of the study cohort.

\begin{tabular}{lccr}
\hline Indicator & $\begin{array}{c}\text { Healthy controls } \\
(\mathrm{n}=76)\end{array}$ & $\begin{array}{c}\text { ACAS patients } \\
(\mathrm{n}=128)\end{array}$ & P-value \\
\hline Age, years & $59.724 \pm 7.652$ & $61.188 \pm 9.267$ & 0.247 \\
Male sex, \% & 64.474 & 67.188 & 0.692 \\
BMI, kg/m & $25.179 \pm 4.488$ & $25.985 \pm 4.607$ & 0.224 \\
TC, mM & $5.150 \pm 1.068$ & $5.817 \pm 1.136$ & $<0.001$ \\
TG, mM & $1.490 \pm 0.283$ & $1.849 \pm 0.394$ & $<0.001$ \\
LDL-C, mM & $2.893 \pm 0.427$ & $3.144 \pm 0.467$ & $<0.001$ \\
HDL-C, mM & $1.065 \pm 0.197$ & $1.019 \pm 0.173$ & 0.081 \\
FPG, mM & $5.098 \pm 0.803$ & $5.912 \pm 1.240$ & $<0.001$ \\
\hline
\end{tabular}

BMI, body mass index; TC, total cholesterol; TG, triglyceride; LDL-C, low-density lipoprotein cholesterol; HDL-C, high-density lipoprotein cholesterol; FPG, fasting plasma glucose; ACAS, asymptomatic carotid artery stenosis.

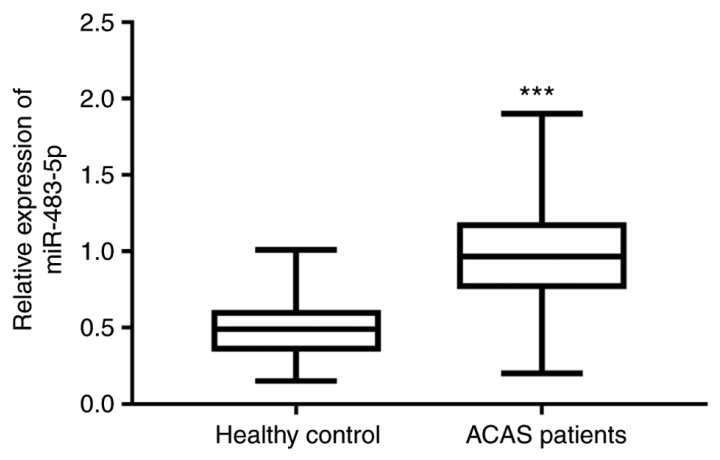

Figure 1. Expression of miR-483-5p in healthy controls and patients with ACAS. ${ }^{* * *} \mathrm{P}<0.001$ vs. healthy control. miR, microRNA; ACAS, asymptomatic carotid artery stenosis.

to be significantly higher in patients with ACAS than that in healthy control $(\mathrm{P}<0.001)$.

Association between serum miR-483-5p and clinical characteristics of patients with ACAS. The relationship between serum miR-483-5p and clinical characteristics of patients with ACAS is provided in Table II. Patients with ACAS were classified into low $(n=60)$ and high $(n=68)$ miR-483-5p expression groups based on the mean value (0.93) of miR-483-5p expression. miR-483-5p expression was indicated to be significantly associated with diabetes $(\mathrm{P}=0.011)$, dyslipidemia $(\mathrm{P}=0.047)$ and the degree of carotid stenosis $(\mathrm{P}=0.006)$. However, there was no significant association between miR-483-5p expression and age, gender, BMI or hypertension (all $\mathrm{P}>0.05$ ).

Diagnostic performance of serum miR-483-5p for ACAS screening. Due to the significantly abnormal expression of serum miR-483-5p in patients with ACAS, an ROC curve was plotted to determine the diagnostic performance of serum miR-483-5p for ACAS screening (Fig. 2). The area under the ROC curve was 0.910 (95\% CI: 87.12-94.94\%) with a sensitivity of $80.5 \%$ and a specificity of $89.5 \%$ at a cutoff value of 0.705 , indicating that miR-483-5p has considerable diagnostic accuracy for ACAS screening.
Serum miR-483-5p predicts cerebrovascular event onset in patients with ACAS. All patients with ACAS were followed up for 5 years, but 16 were lost to follow-up during the 5-year follow-up period due to some of the patients refusing follow-up and could not be contacted. The results of the 5-year follow-up indicated that 30 patients with ACAS developed cerebrovascular events, including 21 TIAs and 9 strokes. Among them, a total of 8 cases in the low-expression group of miR-483-5p developed cerebrovascular events, while 22 cases in the high-expression group of miR-483-5p developed cerebrovascular events. KM curves were then plotted to determine the association between miR-483-5p expression and the onset of cerebrovascular events in patients with ACAS. The results of the KM curve analysis indicated that patients with high expression of miR-483-5p had a lower cumulative proportion of disease-free subjects compared with patients with low miR-483-5p expression (log-rank $\mathrm{P}=0.011$; Fig. 3 ). The results of the Cox regression analysis indicated that miR-483-5p was independently associated with the occurrence of cerebrovascular events $(\mathrm{P}=0.030$; Table III). The above results indicated that miR-483-5p may be used as an independent prognostic marker for predicting cerebrovascular events in patients with ACAS.

\section{Discussion}

A large number of studies have indicated that miRNAs have key roles in the occurrence and development of cerebrovascular diseases, such as miR-9-5p (20) and miR-183-5p (21). In addition, functional miRNAs associated with disease progression are also deregulated in ACAS. For instance, miR-106b-5p (13) and miR-186-5p (22) have been indicated to be abnormal in ACAS and associated with ACAS progression. Yan et al (23) determined that the levels of serum miR-503-5p in patients with ACAS were significantly reduced compared with those in healthy individuals and that miR-503-5p was able to improve CAS. Thus, abnormal miRNA expression is closely associated with the progression of ACAS. However, the relationship between miR-483-5p expression and ACAS had remained to be determined. CAS is generally considered the result of atherosclerosis and plaque rupture (24). A study 
Table II. Association between serum miR-483-5p and the clinical characteristics of patients with asymptomatic carotid artery stenosis.

\begin{tabular}{|c|c|c|c|c|}
\hline \multirow[b]{2}{*}{ Parameter } & \multirow{2}{*}{$\begin{array}{l}\text { Total no. } \\
(n=128)\end{array}$} & \multicolumn{2}{|c|}{ miR-483-5p expression } & \multirow[b]{2}{*}{ P-value } \\
\hline & & Low $(\mathrm{n}=60)$ & High $(n=68)$ & \\
\hline Age, years & & & & 0.855 \\
\hline$<60$ & 48 & 22 & 26 & \\
\hline$\geq 60$ & 80 & 38 & 42 & \\
\hline Sex & & & & 0.383 \\
\hline Female & 42 & 22 & 20 & \\
\hline Male & 86 & 38 & 48 & \\
\hline $\mathrm{BMI}, \mathrm{kg} / \mathrm{m}^{2}$ & & & & 0.390 \\
\hline$<25$ & 40 & 21 & 19 & \\
\hline$\geq 25$ & 88 & 39 & 49 & \\
\hline Diabetes & & & & 0.011 \\
\hline Negative & 68 & 39 & 29 & \\
\hline Positive & 60 & 21 & 39 & \\
\hline Hypertension & & & & 0.532 \\
\hline Negative & 56 & 28 & 28 & \\
\hline Positive & 72 & 32 & 40 & \\
\hline Dyslipidemia & & & & 0.047 \\
\hline Negative & 67 & 37 & 30 & \\
\hline Positive & 61 & 23 & 38 & \\
\hline Degree of carotid stenosis & & & & 0.006 \\
\hline$<70 \%$ & 56 & 34 & 22 & \\
\hline$\geq 70 \%$ & 72 & 26 & 46 & \\
\hline
\end{tabular}

BMI, body mass index.

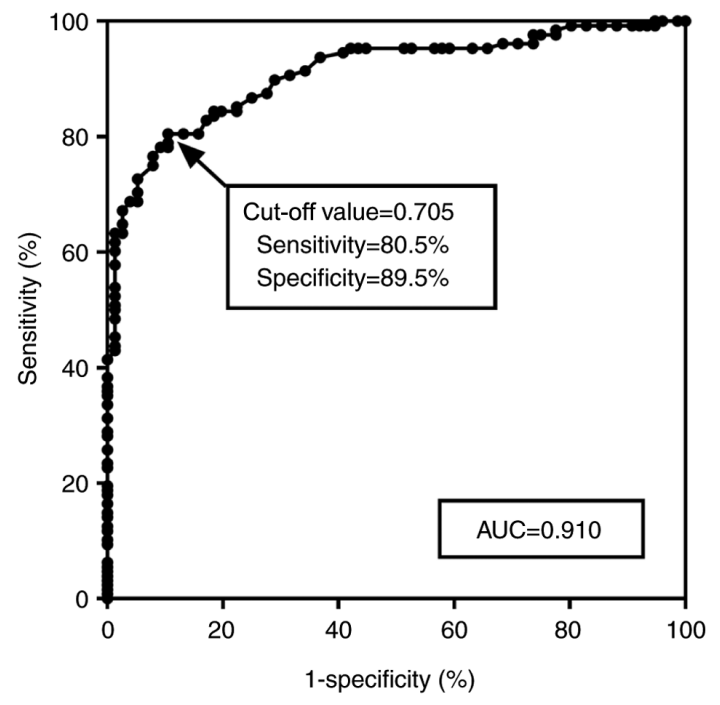

Figure 2. ROC curve for the diagnostic value of serum miR-483-5p levels for screening patients with asymptomatic carotid artery stenosis. The AUC was 0.910 at the cut-off value of 0.705 . ROC, receiver operating characteristic; AUC, area under the ROC curve.

by Li et al (25) reported that miR-483-5p may be a biomarker for the early identification of plaque rupture, suggesting the potential function of miR-483-5p in CAS. In addition, significant correlations of miR-483-5p with obesity and diabetes

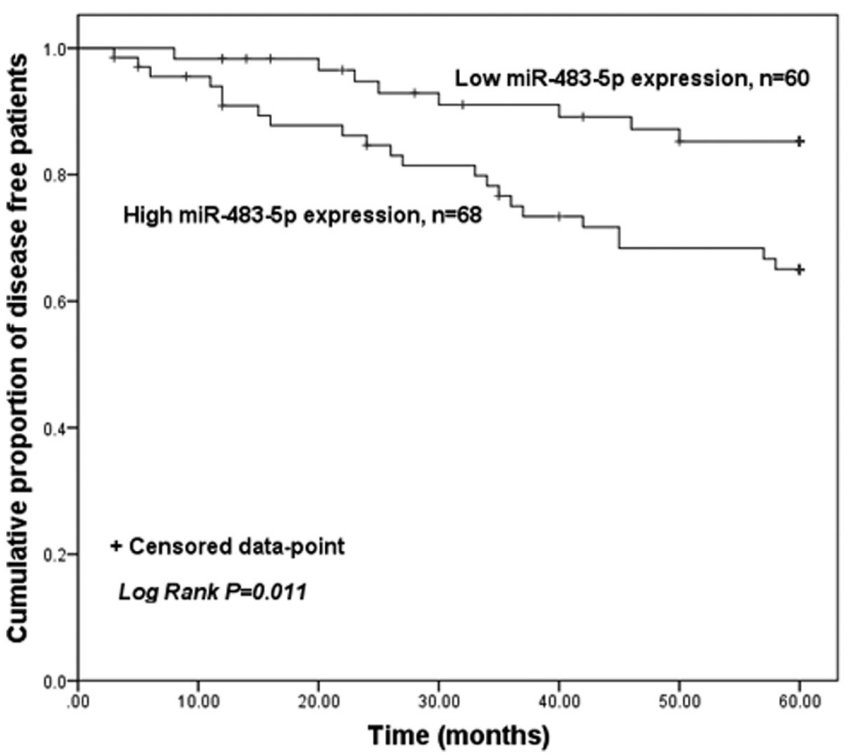

Figure 3. Kaplan-Meier curves to evaluate the onset of a cerebrovascular events in patients with asymptomatic carotid artery stenosis with different miR-483-5p expression (log-rank $\mathrm{P}=0.011$ ). $\mathrm{miR}$, microRNA.

mellitus (risk factors associated with the development of CAS) have been determined (17). The present study indicated that miR-483-5p expression was significantly increased in patients 
Table III. Multivariate Cox regression analysis of factors influencing the risk of a cerebrovascular event in patients with asymptomatic carotid artery stenosis.

\begin{tabular}{lccr}
\hline Variables & HR & $95 \%$ CI & P-value \\
\hline Age, years ( $\geq 60$ vs. $<60)$ & 1.460 & $0.659-3.234$ & 0.352 \\
Sex (males vs. females) & 3.076 & $1.059-8.933$ & 0.039 \\
BMI, $\mathrm{kg} / \mathrm{m}^{2}(\geq 25$ vs. $<25$ ) & 1.495 & $0.638-3.500$ & 0.354 \\
Diabetes (positive vs. negative) & 1.308 & $0.616-2.778$ & 0.484 \\
Hypertension (positive vs. negative) & 1.006 & $0.485-2.089$ & 0.987 \\
Dyslipidemia (positive vs. negative) & 1.545 & $0.726-3.287$ & 0.258 \\
Degree of carotid stenosis $(\geq 70 \%$ vs. $<70 \%)$ & 1.645 & $0.726-3.728$ & 0.233 \\
miR-483-5p (high vs. low) & 2.670 & $1.099-6.484$ & 0.030 \\
\hline
\end{tabular}

${ }^{a}$ High and low miR-483-5p expression is classified based on the mean value (0.93) of miR-483-5p expression. BMI, body mass index; miR-483-5p, microRNA-483-5p; HR, hazard ratio.

with ACAS compared with that in healthy controls. In addition, an association analysis between serum miR-483-5p and clinical characteristics of patients with ACSA revealed that serum miR-483-5p was significantly associated with the degree of carotid stenosis. Furthermore, miR-483-5p has been reported to be involved in other diseases, such as prostate cancer (26), hepatocellular carcinoma (27) and chronic obstructive pulmonary disease (28). Therefore, serum miR-483-5p may be involved in the development of ACAS.

miRNAs may be easily detected from bodily fluids, such as serum and tears, and are stable; thus, they may be utilized as potential biomarkers for various diseases $(29,30)$. For instance, Jia and Liu (31) determined that miR-223 is a biomarker for Alzheimer's disease. Furthermore, serum miR-300 may serve as a biomarker for the diagnosis and prognosis of osteosarcoma (32). A study by Kong et al (33) indicated that serum miR-25 may serve as a diagnostic and prognostic biomarker for human gastric cancer. Of note, numerous studies have reported the diagnostic value of serum miRNAs for ACAS, such as miR-503-5p (23), miR-92a (14) and miR-106b-5p (13). Given the abnormal expression of serum miR-483-5p in patients with ACAS, a ROC curve based on serum miR-483-5p was constructed to evaluate the diagnostic value of miR-483-5p for ACAS. The results suggested that serum miR-483-5p may serve as a promising diagnostic biomarker for distinguishing patients with ACAS from healthy controls. In addition, miRNAs have been determined to have predictive value for cerebrovascular event occurrence. For instance, Chen et al (14) reported that miR-92a may be a potential risk factor for the future onset of cerebrovascular events (including 20 cases of TIA and 8 cases of stroke; study cohort size, 122). A study by Lv et al (22) determined that miR-186-5p was able to predict the occurrence of future cerebral ischemic events (including 19 TIAs and 5 strokes; study cohort size, 67). Serum miR-106b-5p predicted the occurrence of cerebral ischemic events (13). From the above studies, it was indicated that TIA and stroke are the most common cerebrovascular events in ACAS and TIA may occur more frequently than stroke in patients with ACAS. To expand the repertoire of miRNAs with predictive value for the occurrence of cerebrovascular events, the present study further evaluated the predictive value of miR-483-5p for the occurrence of cerebrovascular events in patients with ACAS. It was observed that 30 patients with ACAS developed cerebrovascular events, including 21 cases of TIA and 9 cases of stroke. The results of the KM survival analysis and Cox regression analysis indicated that miR-483-5p may be used as an independent prognostic marker for predicting the occurrence of cerebrovascular events in patients with ACAS. In addition, it should be noted that the diagnostic and prognostic value of serum miR-483-5p for other diseases have been recognized. For instance, serum miR-483-5p was reported as a novel diagnostic and prognostic biomarker for patients with oral squamous cell carcinoma (34). Another study reported that miR-483-5p had a highly specific prognostic value for the clinical outcome of patients with ACC (35). Qu et al (36) indicated that miR-483-5p may be a biomarker for the diagnosis of myeloma and a predictor of survival for patients with myeloma. Thus, it was considered that serum miR-483-5p may be a useful clinical tool for diagnosing ACAS and predicting the occurrence of cerebrovascular events.

The analysis of clinical characteristics of patients with ACSA in different miR-483-5p expression groups revealed that miR-483-5p expression levels were significantly associated with diabetes and dyslipidemia. Diabetes is a risk factor for atherosclerosis and may worsen the degree of carotid stenosis (37). A recent study suggested that miR-483-5p is independently associated with new-onset diabetes mellitus and cardiovascular disease (17). Besides, it is well known that dyslipidemia is a risk factor for the development of CAS (38). The present study demonstrated that miR-483-5p expression levels were significantly associated with dyslipidemia. From the above findings, it was concluded that miR-483-5p may be involved in the development of ACAS by regulating diabetes and dyslipidemia. Furthermore, miR-483-5p was indicated to decrease hypercholesterolemia by targeting proprotein convertase subtilisin/kexin type 9 (PCSK9) (39). Thus, the underlying mechanism of miR-483-5p may be the regulation of high cholesterol by targeting PCSK 9 to affect the development of ACAS. However, further studies are required to explore the underlying mechanisms of the role of miR-483-5p in the development of ACAS. Therefore, a limitation of the 
present study is the lack of a mechanistic analysis, which will be the focus of future research by our group. Another limitation is that the study population was small and a larger study population is required for further study.

In conclusion, the present study indicated that miR-483-5p expression is significantly increased in patients with ACAS. The aberrant expression of miR-483-5p in patients with ACAS may be a candidate biomarker for ACAS diagnosis and the prediction of cerebrovascular event occurrence.

\section{Acknowledgements}

Not applicable.

\section{Funding}

The present study was funded by a Project of Weifang Science and Technology Development (grant no. 2018YX054) and a Scientific Project of Weifang Health and Family Planning Commission (grant no. wfwsjs2018133).

\section{Availability of data and materials}

The datasets used and/or analyzed during the current study are available from the corresponding author on reasonable request.

\section{Authors' contributions}

RL and LJ designed the study and conducted the clinical studies. LJ and XW analyzed data, wrote and revised the manuscript. RL and LJ can confirm the authenticity of the raw data. All authors read and approved the final manuscript.

\section{Ethics approval and consent to participate}

A signed written informed consent was obtained from each patient and the experimental procedures were all in accordance with the guideline of the Ethics Committee of Weifang Hospital of Traditional Chinese Medicine (Weifang, China).

\section{Patient consent for publication}

Not applicable.

\section{Competing interests}

The authors declare that they have no competing interests.

\section{References}

1. Aday AW and Beckman JA: Medical management of asymptomatic carotid artery stenosis. Prog Cardiovasc Dis 59: 585-590, 2017.

2. Wasserman BA, Haacke EM and Li D: Carotid plaque formation and its evaluation with angiography, ultrasound, and MR angiography. J Magn Reson Imaging 4: 515-527, 1994.

3. Coutts SB: Diagnosis and management of transient ischemic attack. Continuum (Minneap Minn) 23: 82-92, 2017.

4. Halliday A, Mansfield A, Marro J, Peto C, Peto R, Potter J and Thomas D; MRC Asymptomatic Carotid Surgery Trial (ACST) Collaborative Group: Prevention of disabling and fatal strokes by successful carotid endarterectomy in patients without recent neurological symptoms: Randomised controlled trial. Lancet 363 $1491-1502,2004$
5. O'Brien M and Chandra A: Carotid revascularization: Risks and benefits. Vasc Health Risk Manag 10: 403-416, 2014.

6. ACST-2 Collaborative Group; Halliday A, Bulbulia R, Gray W Naughten A, den Hartog A, Delmestri A, Wallis C, le Conte S and Macdonald S: Status update and interim results from the asymptomatic carotid surgery trial-2 (ACST-2). Eur J Vasc Endovasc Surg 46: 510-518, 2013.

7. Abbott AL: Medical (nonsurgical) intervention alone is now best for prevention of stroke associated with asymptomatic severe carotid stenosis: Results of a systematic review and analysis. Stroke 40: e573-e583, 2009.

8. Jiang W, Li T, Wang J, Jiao R, Shi X, Huang X and Ji G: miR-140-3p suppresses cell growth and induces apoptosis in colorectal cancer by targeting PD-L1. Onco Targets Ther 12: 10275-10285, 2019.

9. Ow SH, Chua PJ and Bay BH: miR-149 as a potential molecular target for cancer. Curr Med Chem 25: 1046-1054, 2018.

10. Gupta P, Bhattacharjee S, Sharma AR, Sharma G, Lee SS and Chakraborty C: miRNAs in Alzheimer Disease-A therapeutic perspective. Curr Alzheimer Res 14: 1198-1206, 2017.

11. Luan X, Zhou X, Naqvi A, Francis M, Foyle D, Nares S and Diekwisch TGH: MicroRNAs and immunity in periodontal health and disease. Int J Oral Sci 10: 24, 2018.

12. Navickas R, Gal D, Laucevicius A, Taparauskaite A, Zdanyte M and Holvoet P: Identifying circulating microRNAs as biomarkers of cardiovascular disease: A systematic review. Cardiovasc Res 111: 322-337, 2016.

13. Zhang T, Chen Z, Yang X, Fu R, Wang J and Xu H: Circulating miR-106b-5p serves as a diagnostic biomarker for asymptomatic carotid artery stenosis and predicts the occurrence of cerebral ischemic events. Vasc Med 25: 436-442, 2020.

14. Chen G, Gao J, Sheng Y, Han X, Ji X, Zhao M and Wu J: Diagnostic value of miR-92a in asymptomatic carotid artery stenosis patients and its ability to predict cerebrovascular events. Diagn Pathol 15: 74, 2020.

15. Qiao Y, Ma N, Wang X, Hui Y, Li F, Xiang Y, Zhou J, Zou C, Jin J, Lv G, et al: MiR-483-5p controls angiogenesis in vitro and targets serum response factor. FEBS Lett 585: 3095-3100, 2011.

16. Jia L, Hao F, Wang W and Qu Y: Circulating miR-145 is associated with plasma high-sensitivity C-reactive protein in acute ischemic stroke patients. Cell Biochem Funct 33: 314-319, 2015.

17. Gallo W, Esguerra JLS, Eliasson L and Melander O: miR-483-5p associates with obesity and insulin resistance and independently associates with new onset diabetes mellitus and cardiovascular disease. PLoS One 13: e0206974, 2018.

18. Powers WJ, Derdeyn CP, Biller J, Coffey CS, Hoh BL, Jauch EC, Johnston KC, Johnston SC, Khalessi AA, Kidwell CS, et al: 2015 American Heart Association/American Stroke Association Focused Update of the 2013 guidelines for the early management of patients with acute ischemic stroke regarding endovascular treatment: A guideline for healthcare professionals from the American Heart Association/American stroke association. Stroke 46: 3020-3035, 2015.

19. Schmittgen TD and Livak KJ: Analyzing real-time PCR data by the comparative C(T) method. Nat Protoc 3: 1101-1108, 2008

20. Chi L, Jiao D, Nan G, Yuan H, Shen J and Gao Y: miR-9-5p attenuates ischemic stroke through targeting ERMP1-mediated endoplasmic reticulum stress. Acta Histochem 121: 151438, 2019.

21. Zhu L, Zhou X, Li S, Liu J, Yang J, Fan X and Zhou S: miR1835p attenuates cerebral ischemia injury by negatively regulating PTEN. Mol Med Rep 22: 3944-3954, 2020.

22. Lv W, Zhang T, Zhao H, He S, Li B, Gao Y and Pan W: Diagnostic value of miR-186-5p for carotid artery stenosis and its predictive significance for future cerebral ischemic event. Diagn Pathol 15: $101,2020$.

23. Yan Z, Wang H, Liang J, Li Y and Li X: MicroRNA-503-5p improves carotid artery stenosis by inhibiting the proliferation of vascular smooth muscle cells. Exp Ther Med 20: 85, 2020.

24. Meschia JF, Klaas JP, Brown RD Jr and Brott TG: Evaluation and management of atherosclerotic carotid stenosis. Mayo Clin Proc 92: 1144-1157, 2017.

25. Li S, Lee C, Song J, Lu C, Liu J, Cui Y, Liang H, Cao C, Zhang F and Chen H: Circulating microRNAs as potential biomarkers for coronary plaque rupture. Oncotarget 8: 48145-48156, 2017.

26. Yang ZG, Ma XD, He ZH and Guo YX: miR-483-5p promotes prostate cancer cell proliferation and invasion by targeting RBM5. Int Braz J Urol 43: 1060-1067, 2017.

27. Tang S, Chen Y, Feng S, Yi T, Liu X, Li Q, Liu Z, Zhu C, Hu J, $\mathrm{Yu}$ X, et al: MiR-483-5p promotes IGF-II transcription and is associated with poor prognosis of hepatocellular carcinoma. Oncotarget 8: 99871-99888, 2017. 
28. Shen Z, Tang W, Guo J and Sun S: miR-483-5p plays a protective role in chronic obstructive pulmonary disease. Int J Mol Med 40: 193-200, 2017.

29. To KK, Tong CW, Wu M and Cho WC: MicroRNAs in the prognosis and therapy of colorectal cancer: From bench to bedside. World J Gastroenterol 24: 2949-2973, 2018

30. Benz F, Roy S, Trautwein C, Roderburg C and Luedde T: Circulating MicroRNAs as biomarkers for sepsis. Int J Mol Sci 17: 78, 2016

31. Jia LH and Liu YN: Downregulated serum miR-223 servers as biomarker in Alzheimer's disease. Cell Biochem Funct 34: 233-237, 2016

32. Liu JD, Xin Q, Tao CS, Sun PF, Xu P, Wu B, Qu L and Li SZ: Serum miR-300 as a diagnostic and prognostic biomarker in osteosarcoma. Oncol Lett 12: 3912-3918, 2016

33. Kong Y, Ning L, Qiu F, Yu Q and Cao B: Clinical significance of serum miR-25 as a diagnostic and prognostic biomarker in human gastric cancer. Cancer Biomark 24: 477-483, 2019.

34. Xu H, Yang Y, Zhao H, Yang X, Luo Y, Ren Y, Liu W and Li N Serum miR-483-5p: A novel diagnostic and prognostic biomarker for patients with oral squamous cell carcinoma. Tumour Biol 37: 447-453, 2016.

35. Chabre O, Libe R, Assie G, Barreau O, Bertherat J, Bertagna X, Feige JJ and Cherradi N: Serum miR-483-5p and miR-195 are predictive of recurrence risk in adrenocortical cancer patients. Endocr Relat Cancer 20: 579-594, 2013.
36. Qu X, Zhao M, Wu S, Yu W, Xu J, Xu J, Li J and Chen L: Circulating microRNA 483-5p as a novel biomarker for diagnosis survival prediction in multiple myeloma. Med Oncol 31: 219, 2014.

37. Wajima D, Nakagawa I, Takamura Y, Aketa S, Yonezawa T and Nakase H: Carotid artery stenosis is exacerbated in spontaneously obese model rats with diabetes. J Atheroscler Thromb 21: 1253-1259, 2014.

38. Vranic H, Hadzimehmedagic A, Haxibeqiri-Karabdic I, Mujacic E and Djedovic M: Critical carotid artery stenosis in coronary and non-coronary patients-frequency of risk factors. Med Arch 71: 110-114, 2017.

39. Dong J, He M, Li J, Pessentheiner A, Wang C, Zhang J, Sun Y, Wang WT, Zhang Y, Liu J, et al: microRNA-483 ameliorates hypercholesterolemia by inhibiting PCSK9 production. JCI Insight 5: e143812, 2020.

This work is licensed under a Creative Commons Attribution-NonCommercial-NoDerivatives 4.0 International (CC BY-NC-ND 4.0) License. 